\title{
Medaka fish exhibits longevity gender gap, a natural drop in estrogen and telomere shortening during aging: a unique model for studying sex-dependent longevity
}

\author{
Singaram Gopalakrishnan, Napo KM Cheung, Bill WP Yip and Doris WT Au*
}

\begin{abstract}
Introduction: Females having a longer telomere and lifespan than males have been documented in many animals. Such linkage however has never been reported in fish. Progressive shortening of telomere length is an important aging mechanism. Mounting in vitro evidence has shown that telomere shortening beyond a critical length triggered replicative senescence or cell death. Estrogen has been postulated as a key factor contributing to maintenance of telomere and sex-dependent longevity in animals. This postulation remains unproven due to the lack of a suitable animal system for testing. Here, we introduce a teleost model, the Japanese medaka Oryzias latipes, which shows promise for research into the molecular mechanism(s) controlling sex difference in aging.

Results: Using the medaka, we demonstrate for the first time in teleost that (i) sex differences (female $>$ male) in telomere length and longevity also exist in fish, and (ii) a natural, 'menopause'-like decline of plasma estrogen was evident in females during aging. Estrogen levels significantly correlated with telomerase activity as well as telomere length in female organs (not in males), suggesting estrogen could modulate telomere length via telomerase activation in a sex -specific manner. A hypothetical in vivo 'critical' terminal restriction fragment (TRF, representing telomere) length of approximately $4 \mathrm{~kb}$ was deduced in medaka liver for prediction of organismal mortality, which is highly comparable with that for human cells. An age conversion model was also established to enable age translation between medaka (in months) and human (in years). These novel tools are useful for future research on comparative biology of aging using medaka.

Conclusion: The striking similarity in estrogen profile between aging female O. latipes and women enables studying the influence of "postmenopausal" decline of estrogen on telomere and longevity without the need of invasive ovariectomy. Medaka fish is advantageous for studying the direct effect of increased estrogen on telomere length and longevity without the breast cancer complications reported in rodents. The findings strongly support the notion that $\mathrm{O}$. latipes is a unique non-mammalian model for validation of estrogenic influence on telomere and longevity in vertebrates. This laboratory model fish is of potential significance for deciphering the ostensibly conserved mechanism(s) of sex-associated longevity in vertebrates.
\end{abstract}

Keywords: Lifespan, Aging, Telomerase and telomere, Estrogen profile, Sex difference and medaka O. latipes

\footnotetext{
* Correspondence: bhdwtau@cityu.edu.hk

State Key Laboratory in Marine Pollution, Department of Biology and

Chemistry, City University of Hong Kong, 83 Tat Chee Avenue, Kowloon, Hong Kong SAR
}

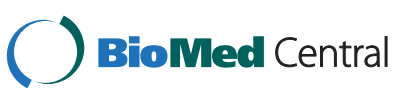

(c) 2013 Gopalakrishnan et al.; licensee BioMed Central Ltd. This is an Open Access article distributed under the terms of the Creative Commons Attribution License (http://creativecommons.org/licenses/by/2.0), which permits unrestricted use, distribution, and reproduction in any medium, provided the original work is properly cited. The Creative Commons Public Domain Dedication waiver (http://creativecommons.org/publicdomain/zero/1.0/) applies to the data made available in this article, unless otherwise stated. 


\section{Introduction}

Longevity gender gap (LGG) is the longevity difference between the two sexes. Many animals exhibit a longer lifespan in females than males (Table 1). Several theories have been proposed to explain the possible cause(s) of LGG (females > males) [1]. Among them, sex differences in telomere length (longer in females) and estrogen (higher in female) have been given the most attention, particularly in mammalian studies [2-5]. Telomeres are DNA capping structures that protect chromosome ends from recombination and fusion, maintaining genomic stability [6]. Telomeres shorten with age due to inefficiency in DNA replication (a.k.a. end-replication problem) [7]. Shortened telomere length (TL) below threshold level induces cellular senescence or cell death [8]. Progressive erosion of telomere length is an important aging process, which is well recognized by extensive in vitro and in vivo studies on mammals $[4,9]$. The enzyme telomerase promotes telomeric repair and reduces telomere erosion by adding conserved repeats of TTAGGG to chromosomal ends [10]. In vitro mammalian studies demonstrated that estrogen can stimulate telomerase activity via estrogenreceptor-mediated transcription and post-translational activation of TERT (telomerase reverse transcriptase; the catalytic unit of telomerase) [11,12]. Estrogen is synthesized in all vertebrates and some invertebrates $[13,14]$, therefore estrogen-mediated telomerase activation and telomere maintenance are likely conserved in animals.

Despite estrogen being described as a key factor contributing to the observed sex differences in telomere length (female $>$ male) and longevity in animals, this hypothesis has never been validated using a suitable model system. The common animal models employed for aging studies, Caenorhabditis elegans and Drosophila melanogaster, have postmitotic cells predominantly in the somatic tissues of adult, making it unfeasible to investigate telomere-associated replicative senescence [46].
The conventional rodent models are not desirable for telomere- and estrogen- related aging studies. This is because telomeres of inbred rodents are extraordinarily long [47], making it difficult to study the effects of telomere erosion on aging and LGG in either short-term experiments or within a single generation. Moreover, the increased risk of breast and ovarian cancer development upon estrogen administration in rodents [48,49] complicates the study of the direct effects of estrogen on telomere length and longevity in vivo.

The small sized fish, Japanese medaka (Oryzias latipes), shares similar estrogen biology with mammals [50]. They undergo gradual senescence, increasing mortality rate with age [51-53] and progressive telomere shortening in most organs as it ages [54]. Our preliminary study further reveals female medaka live longer than the males. However, sex difference in telomere has never been reported in $O$. latipes or any teleost [45]. Using the medaka O. latipes as a model, the present study was designed to answer the fundamental question "Do sex differences (female $>$ male) in both telomere length and longevity exist in fish?" Supported by the positive outcomes, we further examined age-associated changes in sex hormones, telomerase activity and telomere (length and attrition rate) in the liver (organ common in vertebrates) and gill (organ unique in fish), with an attempt to infer the mechanistic relationship among estrogen (sex), telomere and longevity in O. latipes. A theoretical 'critical' telomere length was derived in medaka liver for prediction of organismal senescence and mortality. Our findings also highlight a striking similarity between female $O$. latipes and women regarding their age-associated change in estrogen profile and telomere dynamic. The medaka $O$. latipes is therefore unique for studying the mechanisms of estrogenic (sex) influence on longevity in vertebrates, particularly human. To facilitate future research in this direction, we put forward a mathematical model that permits age conversion between medaka and human. Evidences provided herein strongly

Table 1 The existence of longevity gender gap (females living longer than males) in different animal taxa

\begin{tabular}{|c|c|c|c|}
\hline & Taxa & & References \\
\hline \multirow[t]{8}{*}{ Vertebrates } & Mammals & Human & {$[2,3,15-17]$} \\
\hline & & Chimpanzees & {$[18,19]$} \\
\hline & & Gorillas, Orangutans, gibbons, spider monkeys and sifakas & {$[18,20]$} \\
\hline & & Rat & {$[15-17,21]$} \\
\hline & & Soay sheep, pilot whales and killer whales & {$[22,23]$} \\
\hline & Reptiles & Lizard & {$[24-26]$} \\
\hline & Amphibians & Frogs and newts & {$[27-30]$} \\
\hline & Fishes & Spiny dogfish; mosquitofish and scaldfish & {$[31-33]$} \\
\hline Invertebrates & Arthropods and annelids & $\begin{array}{l}\text { Fruit flies, medflies, butterflies, mosquitoes, seed beetles, ants, bees, tarantulas, tea } \\
\text { red spiders, copepods and the giant kidney-worms in the maned wolf }\end{array}$ & {$[34-44]$} \\
\hline
\end{tabular}

Footnote: An alternative form of longevity gender gap, i.e. males living longer than females, is also exhibited in a few animal taxa, especially the Aves [45]. 
support that medaka fish is desirable for research in comparative biology of aging, unraveling the evidently conserved mechanism(s) of sex-dependent longevity in vertebrates.

\section{Results}

\section{Sex-dependent lifespan of medaka}

Male and female medaka reared under stable laboratory condition displayed significantly different survival profiles (Figure 1; logrank test: $\chi^{2}=6.51, d f=1, \mathrm{~N}=3117$, $p=0.011$ ). Major discrepancy in survival probability between the two sexes was observed at $4-15$ months old (Figure 1). The median life span of the males and females was 13.7 (95\% CI: 13.3 - 14.1) months and 14.6 (95\% CI: 14.3 - 15.2) months, respectively.

\section{Sex-dependent telomere length in the liver and gill of medaka during aging}

Telomere length (TL) represented by the mean terminal restriction fragment size (dubbed 'TRF' hereafter) in both gill and liver was not only age-dependent, but also sex-specific (Figure 2; two-way ANOVA interaction effect; Gill: $F_{3,70}=8.43, p<0.001$; Liver: $F_{3,72}=$ 30.4, $p=0.034)$. Significant difference in TRF between sexes was observed in 'younger' individuals (i.e. gill: 4 months old; liver: 4-8 months old). Whenever significant difference in TRF was detected between two sexes, it was always longer in the females than in males (Figure 2).

\section{Organ-dependent telomere attrition rate in male and female medaka during aging}

The age-associated decline of TRF, as observed in the gill and liver, grossly followed an exponential decay model $\left(R^{2} \geq 0.89\right)$. The rate of telomere attrition in the liver was significantly faster than that in the gill, and the telomere attrition rates were not sex-dependent $(95 \% \mathrm{CI}$ of $\lambda$ : Liver $\hat{\sigma}=1.67-2.55$, ㅇ $=1.75-2.13$; Gill $\hat{\partial}=0.77-1.25$, $=$ $0.80-1.08)$. Besides, TRF in the gill was maintained in medaka from 12 months onwards; whereas in the liver, a continue reduction in TRF was observed across age for both sexes (Figure 2: top vs bottom panel).

\section{Sex-dependent telomerase activity in the liver and gill of medaka during aging}

Telomerase activity was sex-specific and age-dependent in both gill (interaction effect: $F_{4,90}=4.15, p=0.004$ ) and liver $\left(F_{4,90}=7.75, p<0.001\right)$. In gill, telomerase activity was significantly higher in females at 8-22 months old than in males of the same ages. By contrast, in the liver, 4-8 months old females showed higher telomerase activity than the male counterparts (Figure 3). Age dependent change in telomerase activity was much more prominent in females than in males (Figure 3: left vs right). In females, telomerase activity peaked in both gill and liver at 8 months old.

\section{Plasma sex hormone levels in male and female medaka during aging}

Statistical significant difference between males and females of the same age was detected in estradiol (E2),

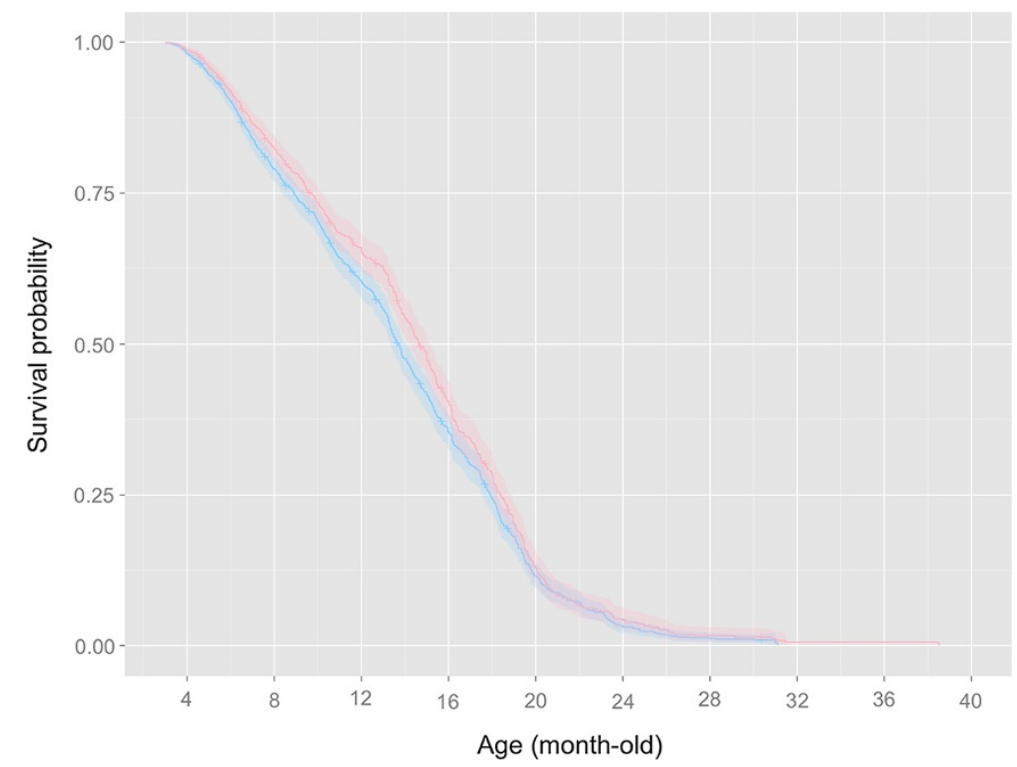

Figure 1 Kaplan-Meier survival curves of the male (blue) and female (pink) O. latipes reared under optimal laboratory conditions (see Material and methods; $\mathbf{N}=\mathbf{3 1 1 7}$ ). Confidence intervals (95\%) of the survival rate are shown as semi-transparent colored bands. The survival probability of females is generally higher than that of males until 16 month-old, note the minor- to non-overlapping confidence intervals. 


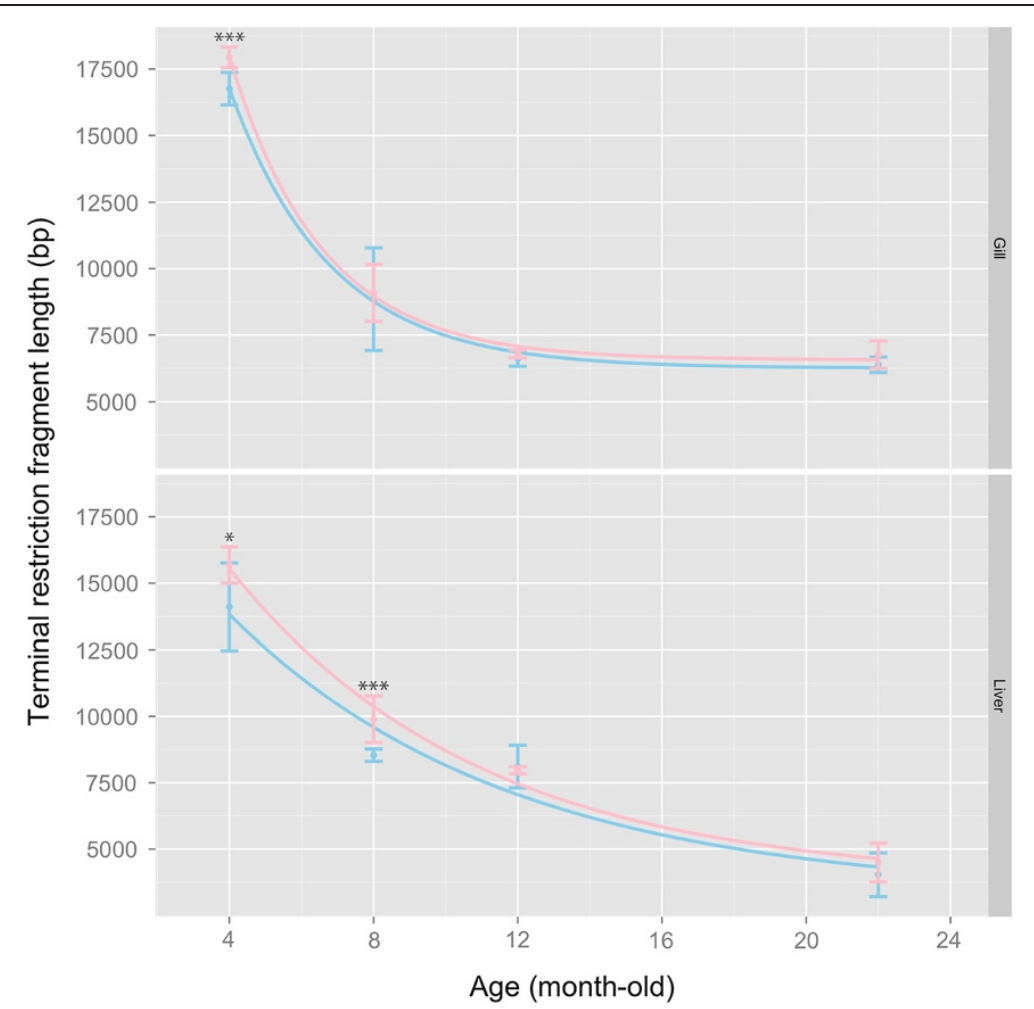

Figure $\mathbf{2}$ Change of telomere length, as represented by the size of terminal restriction fragment (TRF; in bp), in male (blue) and female (pink) O. latipes during aging (upper: gill; lower: liver). Shortening of organ TRF length across age in each sex was modeled by an exponential decay curve (see Materials and Methods). Error bars are standard error of mean (SEM). Asterisk indicates significant difference in TRF length between sexes at specific age: ${ }^{*}=p<0.05,{ }^{* * *}=p<0.001$ after FDR-adjustment.

testosterone $(\mathrm{T})$ and 11-keto-testosterone (11-KT) (all FDR-adjusted $p<0.05)$. Plasma E2 level in the females peaked at 8 months old and sharply declined thereafter, while that in the males was fairly constant. Similarly, plasma $\mathrm{T}$ and 11-KT levels peaked in males at 8 months old and gradually decreased with age, whereas their levels remained fairly stable in females across age. In both sexes, the levels of all three sex hormones in 22 months old $O$. latipes were significantly lower than fish of younger ages $(4-15$ months old) $(p<0.05)$ (Figure 4$)$.

\section{Correlation between sex hormone levels, telomerase activity and telomere length}

The relationships among levels of plasma sex hormones, telomerase activity and TRF were sex-specific and were much more evident in females (Table 2). In both gill and liver of females: (1) plasma E2 level was positively correlated with telomerase activity as well as TRF, and (2) T/11-KT also significantly correlated with TRF. Conversely, in males, (1) neither telomerase activity nor TRF correlated with E2 level, and (2) T/11-KT correlated with TRF in the liver only (not in gill) and at a lower correlation coefficients than that of the females. Spearman's correlation analysis revealed no significant relationship between telomerase activity and TRF organ-wise and sex-wise in $O$. latipes (FDR-adjusted $p>0.05$ ), except in the gill of males $(\rho=0.62$, FDR-adjusted $p<0.001)$.

\section{'Critical' telomere length for medaka liver}

Survival probability of individual fish was linearly related to the hepatic telomere length and reached almost 100\% survival when TRF exceeded $\sim 11 \mathrm{~kb}$ (Figure 5). Extrapolation of the TRF-survival relationship marks $0 \%$ survival probability (i.e. $100 \%$ mortality) when hepatic TRF decline to a mean 'critical' length of $3.8 \mathrm{~kb}$ and this was not sex-dependent ( $\delta: 3.2-4.2 \mathrm{~kb}$; 9 : $3.4-4.4 \mathrm{~kb})$.

\section{Discussion}

\section{Sex differences in telomere length and longevity exist in} fish

This is the first report in teleost that females have a longer telomere length and lifespan than males. Our continued monitoring of over 3000 male and female Japanese medaka reared under optimal laboratory conditions for over three years produced sufficient and reliable data, supporting that a longer life expectancy in female $O$. latipes was concurred with a longer telomere in both the liver and gill. Further examination over a wide spectrum of fish species would be useful to verify the prevalence of these biological phenomena in primitive vertebrates. 


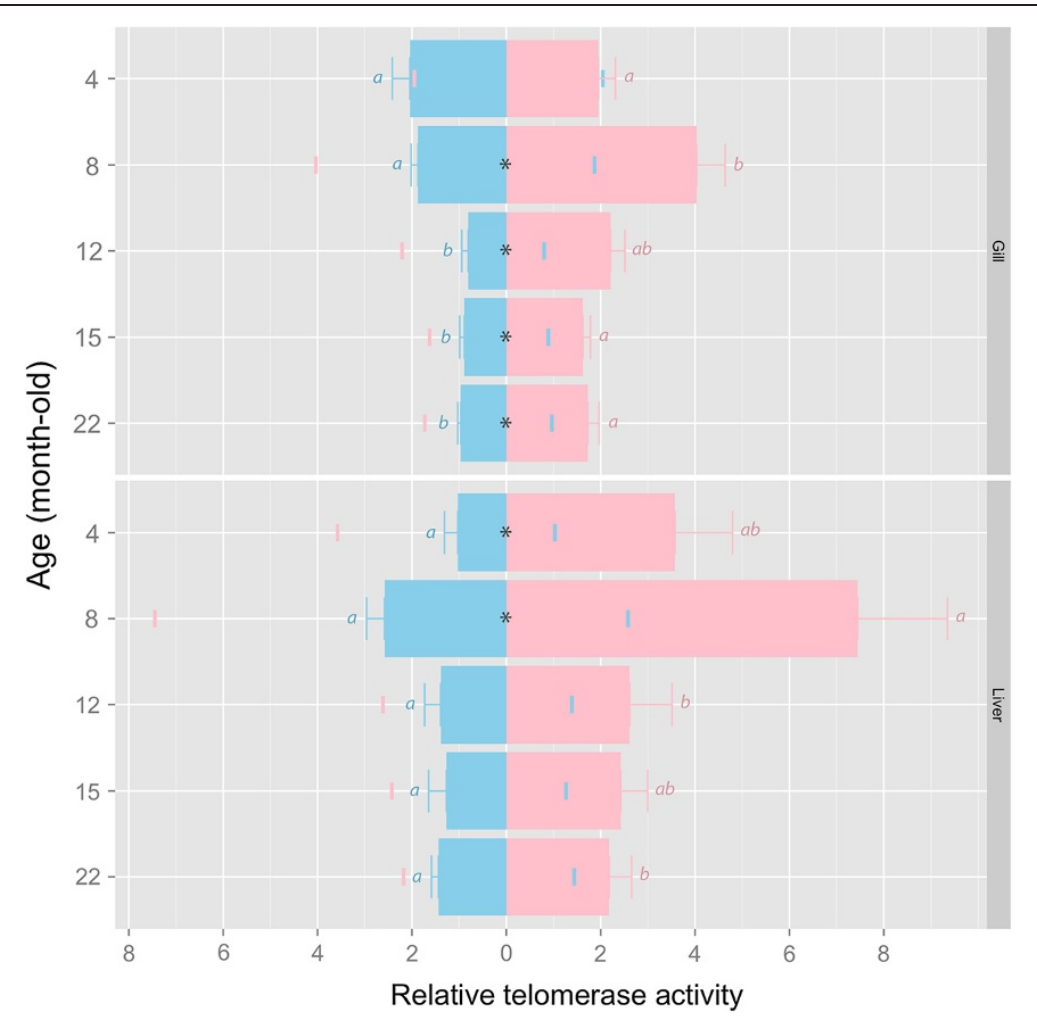

Figure 3 Change of telomerase activity in male (blue) and female (pink) O. latipes during aging (upper: gill; lower: liver). Relative telomerase activity is denoted by the bar length and the colored mark on the opposite side of the origin. Error bars are standard error of mean (SEM). Bars annotated with different alphabets are significantly different. Asterisk $\left(^{*}\right)$ indicates significant sex difference at that particular age (FDR-adjusted $p<0.05$ ).

\section{'Menopause'-like estrogen profile in female Japanese medaka during aging}

Another novel finding arising from this study is the discovery of a natural drop of plasma E2 level in aging female O. latipes: E2 level was high in young females (4 months), peaked at mature females (8 months), followed by a significant decline in senior females (after 12 months). This is similar to the E2 profile from puberty to menopause in women [55]. In rodents, agerelated decline of E2 level is not distinct, therefore ovariectomy is needed to mimic postmenopausal decline of E2 in vivo [56]. This invasive, artificial procedure could disturb body homeostasis, independent of the loss of estrogen [57]. In this regard, the O. latipes is unique over the rodent models for studying the estrogenic influence on telomere in 'postmenopausal' females without the necessity of ovariectomy.

\section{Potential mechanistic relationship between sex hormones, telomerase and telomere in medaka}

Estrogen concentrations in plasma are useful to explain the sex-dependent telomere maintenance in O. latipes: when the females exhibited very high or peaked E2 levels at 4-8 months old, sex difference in telomere length (female $>$ male) was the most distinct; whereas when E2 level declined in senior females, e.g. > 12 months old, such sex difference was correspondingly attenuated. It has been well proven using a variety of human cells [58] that estrogen enhances telomere maintenance by stimulating telomerase activity through EREdependent upregulation of TERT expression $[11,12]$. Ongoing study in our lab indicates the presence of functional EREs in O. latipes TERT promoter, suggesting this estrogen- and telomerase-dependent pathway also exists in O. latipes.

On the other hand, given a very weak link between testosterone/11-keto-testosterone and telomerase activity in both male and female O. latipes (Table 2), the regulatory effect of the two masculine hormones on telomere maintenance is likely independent of telomerase. This seems contradictory to previous in vitro studies showing that androgen could modulate hTERT expression and telomerase activity in prostate and hematopoietic cells [59-61]. The in vivo regulatory effect of androgen on telomerase and telomere maintenance in vertebrates warrants further investigation. 


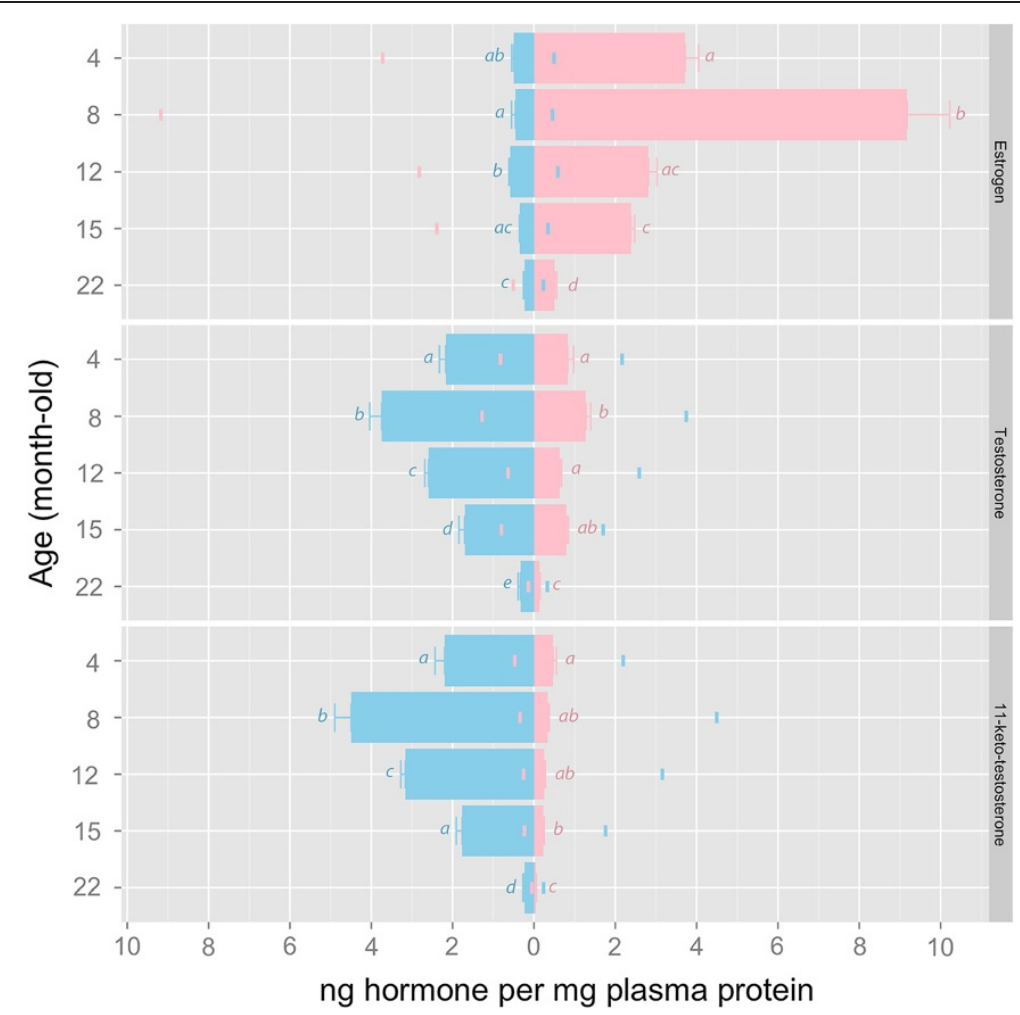

Figure 4 Change of plasma sex hormones level in male (blue) and female (pink) O. latipes during aging (top: estrogen; middle: testosterone; bottom: 11-keto-testosterone). Normalized hormone levels are denoted by the bar length and the colored mark on the opposite side of the origin. Error bars are standard error of mean (SEM). Bars annotated with different letters are significantly different. Statistical significant difference between the two sexes was detected for all three sex hormones at all age groups (all FDR-adjusted $p<0.05$ ).

Organ "critical" telomere length for prediction of survival probability

Earlier studies showed that human cells seldom possess mean TRF length shorter than $4 \mathrm{~kb}$, and any further telomere attrition beyond this "critical" length was correlated with an induction of replicative senescence or cell death in vitro [61-63]. However, there was no report

Table 2 Spearman correlation between sex hormones and relative telomerase activity (TA) as well as telomere length (TL) in Japanese medaka O. latipes

\begin{tabular}{ccccccc}
\hline & & \multicolumn{2}{c}{ Female } & & \multicolumn{2}{c}{ Male } \\
\cline { 7 - 8 } \cline { 7 - 7 } \cline { 6 - 7 } Estrogen & Gill & $0.31^{*}$ & $0.51^{* *}$ & & - & - \\
(E2) & Liver & $0.34^{*}$ & $0.68^{* *}$ & & - & - \\
Testosterone & Gill & - & $0.47^{* *}$ & & $0.41^{*}$ & - \\
(TT) & Liver & - & $0.60^{* * *}$ & & - & $0.41^{*}$ \\
11-keto testosterone & Gill & - & $0.54^{* *}$ & & - & - \\
(11-KT) & Liver & - & $0.71^{* * *}$ & & - & $0.39^{*}$ \\
\hline
\end{tabular}

Numerical figures are Spearman's rank-order correlation coefficients $(\rho)$ that are statistical significant. Asterisks represent FDR-adjusted $p$-values. ${ }^{*} p<0.05$, ${ }^{* *} p<0.01,{ }^{* * *} p<0.001,-:$ not significant. of similar "critical" telomere length in organs in vivo that could link to an induction of organismal senescence or mortality. Based on the temporal profile of telomere attrition in the liver of $O$. latipes, we have deduced a hypothetical in vivo 'critical' TRF length of approx. $4 \mathrm{~kb}$, which is concurred with a zero survival probability of $O$. latipes (Figure 5). This theoretical in vivo 'critical' length in medaka liver is highly comparable to that for human cells, which may suggest senescence is triggered by a common telomere threshold. However, prior to making such a generalization, it is prudent to verify by using a variety of biological systems. Nevertheless, this 'critical TRF' in liver (not sex-dependent) marks a potential association between telomere attrition, organismal senescence and mortality of $O$. latipes. The potential of this 'critical TRF' as a novel molecular biomarker for predicting mortality in $O$. latipes warrants further testing and validation.

Furthermore, we recognize that 'critical TRF' could not be extrapolated for $O$. latipes gill which exhibited a level off of telomere length at old age. This also sheds light on the importance of multiple organ systems approach for a holistic understanding of telomere dynamics and aging progression in animals. 


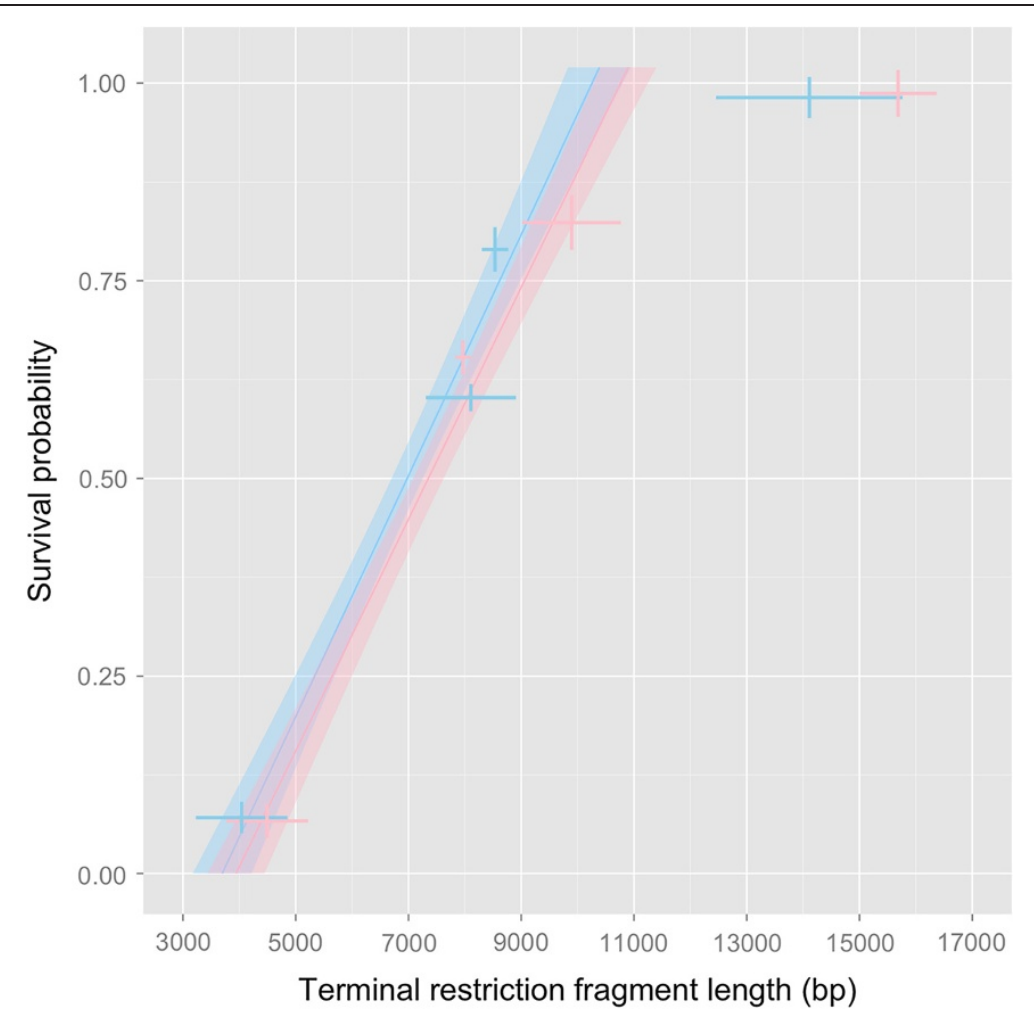

Figure $5 \mathrm{~A}$ prediction model established to forecast survival probability from hepatic telomere length in 0 . latipes. The survival probability is grossly proportional to the hepatic TRF length $\left(R^{2}=0.893\right)$. 'Zero' survival probability of male and female $O$. latipes is equivalent to a 'critical' length of ca. $3.8 \mathrm{kbp}$. Crosses are overlaid error bars ( $95 \% \mathrm{Cl}$ ) for survival probability (vertical) and hepatic telomere length (horizontal).

\section{Age conversion between medaka and human}

Our findings show that the initial TRF length (12 $17 \mathrm{~kb}$ ) of young Japanese medaka (4 months) was much shorter than mice $(100-150 \mathrm{~kb})[64]$ but similar to human $(10-15 \mathrm{~kb})$ [65]. Compare to rodent, the shorter telomere in medaka enables the study of the effects of telomere erosion on aging and LGG in a single generation. Interestingly, despite the similarity in initial and 'critical' TRF length between medaka and human, they exhibit distinct difference in lifespan (median in medaka: 14.3 months; in human, 65 years). The results imply one medaka-month could be equivalent to many humanyears. To facilitate researchers to translate medaka age (in months) to human age (in years), we establish a mathematical model for age conversion (Figure 6) by using three key age hallmarks (sexual maturation age, the median lifespan and the maximum life span) reported for $O$. latipes to plot against the corresponding ages known for humans. Compare with other studies reported for $O$. latipes at these age hallmarks, our data were in line with [66], but different from [54]. This may be due to variation in husbandry conditions (e.g. indoor vs outdoor) in different laboratories. To validate and test the power of this conversion model, we interpolated 8- and 12- months old females, which encompassed a drastic decline in plasma E2 level after the peak at 8 months (Figure 6: highlighted band above the curve) to the corresponding ages in women (Figure 6: highlighted band under the curve). The results were found to be between $46-60$ years old, which fully agree with the menopause age range in women [55]. This age conversion model is reliable and useful for future research on comparative biology of aging.

\section{Summary}

The findings of this study strongly support the notion that $O$. latipes has advantages as a model for research on sex differences in telomere, aging and longevity because it exhibits: (i) longevity gender gap (female $>$ male) and (ii) sex difference in telomere length (female $>$ male). The natural, 'menopause'-like drop of estrogen in females during aging renders medaka a unique model for studying the effect of 'postmenopausal' decline of estrogen on telomere and longevity without the need of invasive ovariectomy as required in rodents. Moreover, the direct effect of increased estrogen on telomere and longevity could be investigated in fish without the complication of breast cancer as reported in rodents. All these evidences highlight the strong potential of O. latipes as a 


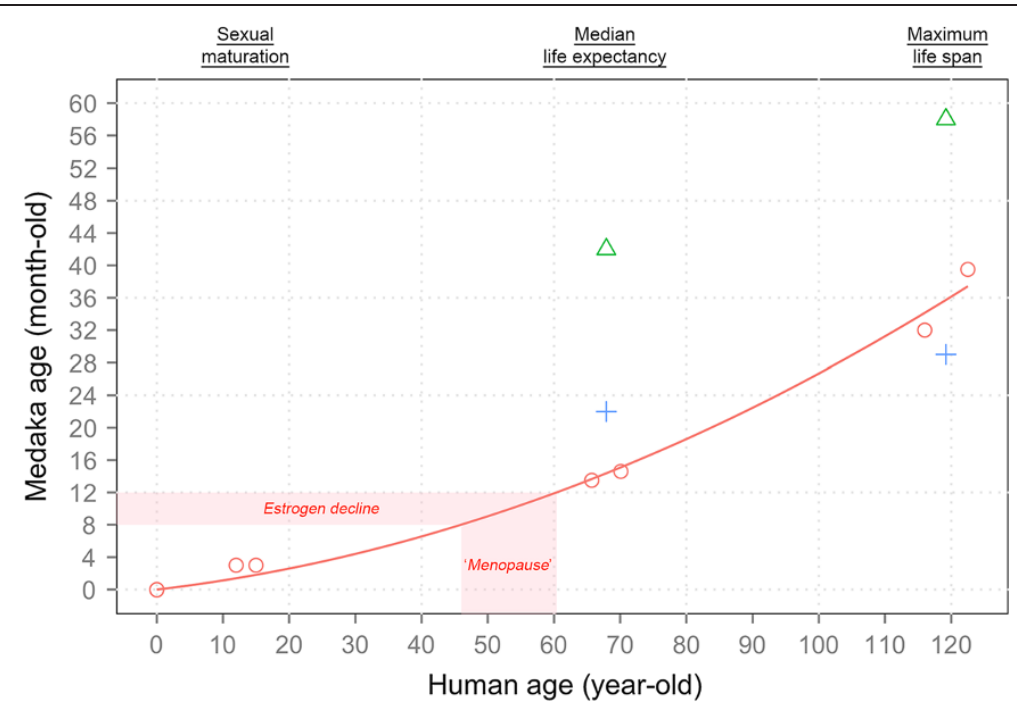

Figure 6 An age conversion model for O. latipes (in months) and human (in years): Age $_{\text {medaka }}=0.0954 \times$ Age $_{\text {human }}+0.0171 \times$ Age $_{\text {human }}^{2}$. Three major hallmarks: sexual maturation, median life expectancy and up-to-date record of maximum lifespan were used for regression. Red circles o represent data from the present study: sexual maturation (O. latipes : ca. 3 month-old; human: 12-15 year-old), median life expectancy (O. latipes: $\hat{\delta}=13.7 \mid \phi=14.6$ months; human: world's average $\hat{\delta}=65.7 \mid q=70.1$ years) and up-to-date record of maximum lifespan $(O$. latipes: $\hat{\delta}=32.0 \mid q=39.5$ months; human: $\hat{\sigma}$ Jiroemon Kimura $=116.1 \mid$ i Jeanne Calment $=122.4$ years $)$. According to the regression curve $\left(R^{2}=0.996\right)$, the occurrence of estrogen decline in the female medaka (between 8 to 12 month-old) in our culture could be interpolated to human age of 46.0 to 60.4 year-old, which is akin to "menopause" in women. Previous aging data from [66] (blue cross + ) and [54] (green triangle $\Delta$ ) were overlaid for reference.

unique non-mammalian model to advance research on estrogen- (sex-), telomere-associated aging and longevity in vertebrates. The in vivo critical length of medaka liver for prediction of organismal mortality and the age conversion model for medaka and human are useful tools for future research on comparative biology of aging using medaka. Overall, evidences provided herein highlight that the teleost $O$. latipes is significant for deciphering the ostensibly conserved mechanism(s) of sex-associated longevity in vertebrates.

\section{Material and methods \\ Medaka culture}

Orange-red, outbred line of Japanese medaka (Oryzias latipes) was originated from the Duke University, Molecular Aquatic Toxicology Laboratory. In 2008, fish were transferred and maintained as a colony in the City University of Hong Kong. Fertilized eggs were collected daily and reared until hatch as described in [67]. Fish that hatched in the same month were grouped as one cohort. Upon sexual maturation (approx. 3 month-old), males and females from the same cohort were randomly paired and transferred to aquarium tanks $(39.5 \mathrm{~L} \times$ $23.5 \mathrm{~W} \times 27.5 \mathrm{H} \mathrm{cm})$ at a density of 15 pairs per tank. The fish were kept in the same set of tanks throughout their whole life.

All fish were kept under static condition of $26 \pm 1^{\circ} \mathrm{C}$, $7.2 \pm 0.2 \mathrm{mg} \mathrm{O}_{2} \mathrm{~L}^{-1}$ and 14:10 hrs light-dark cycle. Half of the tank water is replaced with charcoal-filtered tap water every day. The fish were feed twice daily with Otohime $\beta 1$ (Nisshin Co, Japan) and supplemented freshly hatched brine shrimp (Artemia nauplia) (Ocean Star International Lucky Brand, Utah, USA) 3 days per week. Fish health was closely monitored. Unhealthy fish, usually characterized by lack of appetite, inactivity, loss of golden-red color, hemorrhages, and/or external outgrowth, were promptly isolated into individual glass containers (diameter: $14.5 \mathrm{~cm}$; height: $5 \mathrm{~cm}$ ) containing $500 \mathrm{ml}$ charcoal-filtered tap water. Every quarantined fish was reared under identical condition as described above and would not return to originated tank unless symptoms disappeared ('recovered') for at least 2 weeks. Records of stocking density and daily mortality of males and females in individual tank (hence individual cohort) were curated in SQLite 3 relational database management system (www.sqlite.org). These records were extracted for generating survival profiles and statistics.

\section{Fish sampling}

Healthy fish at five different ages were sampled: 4('young'), 8- ('mature'), 12- ('senior'), 15- ('old') and 22('very old') months. At each time point, 90 fishes (45 males and 45 females) were dissected. Fish were anesthetized in ice-cold aquarium water for $30 \mathrm{~s}$, removed and measured for body length and weight. The fish was kept sedated by gently wrapping with paper towel fully soaked 
with ice-cold aquarium water. A cut was made to tail at 1-2 mm rostral to the caudal fin. Blood was drawn from the cut using P10 micropipette (Eppendorf, Hamburg, Germany) attached with heparinized pipette tips and instantly diluted with $8 \mu \mathrm{L}$ double-distilled water to prevent clotting. The fish was then immediately decapitated. Gill and liver were isolated on ice bed and divided into two equal portions for parallel telomerase activity and telomeric length measurements. Blood and tissue samples from three fish of the same sex were pooled as one replicate (i.e. final sample number $=15$ per sex per time-point). Pooled samples were snap-frozen in liquid nitrogen and stored at $-80^{\circ} \mathrm{C}$ until further processing. Animal handling procedures as mentioned above were accepted by the Animal Ethics Committee, City University of Hong Kong.

\section{Telomere length measurement by Southern blot analysis}

Genomic DNA was extracted from the livers and gills of adult male and female medaka using the DNeasy ${ }^{\circ}$ Blood and Tissue Kit (Qiagen, Hilden, Germany) according to the manufacturer's instructions. For each assay, $3 \mu \mathrm{g}$ genomic DNA was digested to completion with RsaI and HinfI (New England Biolabs, Massachusetts, USA) at $37^{\circ} \mathrm{C}$ overnight. The digested DNA was resolved by electrophoresis on a $1 \%$ agarose gel, run in parallel with a $\lambda$ HindIII/EcoRI molecular marker (Fermentas, Burlington, Canada) and subsequently transferred to Hybond-XL membrane (GE Healthcare, Little Chalfont, United Kingdom) for overnight through capillary transfer. The membrane was saturated with ExpressHyb ${ }^{\mathrm{Tw}} \mathrm{Hy}$ bridization Solution (Clontech, California, USA) at $42^{\circ} \mathrm{C}$ for 30 mins, and hybridized with 100 pmol of DIG-

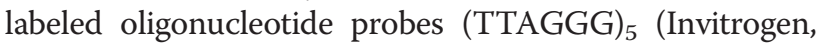
California, USA) in ExpressHyb ${ }^{\text {tu }}$ Hybridization Solution (Clontech Laboratories, California, USA). The DIGlabeling was achieved by the use of DIG Oligonucleotide Tailing Kit, $2^{\text {nd }}$ generation (Roche Applied Science, Penzberg, Germany) according to the manufacturer's instructions. After hybridization, the membrane was washed two times in $2 \mathrm{X} \mathrm{SSC}$ buffer with $0.1 \% \mathrm{SDS}$ at $25^{\circ} \mathrm{C}$ ( 5 mins each) and twice for 15 mins in $0.1 \mathrm{X} \mathrm{SSC}$ with $0.1 \%$ SDS at $42^{\circ} \mathrm{C}$. The washed membrane was blocked in $3 \%$ solution of non-fat milk powder (in phosphate buffered saline, PBS; pH 7.4) for 30 mins at room temperature, then incubated with 1:10000 anti-DIG, APconjugated antibodies (Roche). The membrane was then washed three times in PBS at RT and incubated with CDP-star (Roche) in darkness for 5 mins. Chemiluminescence was imaged by luminiscent image analyzer (Fujifilm LAS 4000, Tokyo, Japan) and saved lossless as TIFF. Telomere length was quantified in ImageJ as terminal restriction fragment (TRF) length following the procedure of [68].
Telomerase activity assays by the real-time quantitative telomeric repeat amplification protocol (RTQ-TRAP)

Frozen tissues samples were thawed on ice bed and lysed in ice-cold CHAPS-containing lysis buffer $(10 \mathrm{mM}$ Tris- $\mathrm{HCl}, \mathrm{pH} 7.5,1 \mathrm{mM} \mathrm{MgCl}$, 1 mM EGTA pH 8.0, $0.5 \%$ CHAPS, $10 \%$ glycerol, $0.1 \mathrm{mM}$ PMSF, $5 \mathrm{mM} \beta-$ mercaptoethanol) (Sigma-Aldrich, Missouri, USA). The lysates were centrifuged at $16000 \times \mathrm{g}, 4^{\circ} \mathrm{C}$ for 30 mins. The supernatants were carefully transferred to new sterilized microfuge tubes. Protein concentration was determined using the Bradford protein assay (Bio-Rad, California, USA), with reference to standard curve constructed from serial dilutions of protein standard (bovine serum albumin, BSA; Sigma-Aldrich).

Telomerase activities in liver and gill samples of medaka were assessed by the RTQ-TRAP assay following the optimized protocol by [69]. Briefly, $40 \mathrm{ng}$ of protein extract was added to a $25 \mu \mathrm{L}$ reaction mixture of TRAP buffer [20 mM Tris- $\mathrm{HCl}(\mathrm{pH} 8.3), 63 \mathrm{mM} \mathrm{KCl}, 3.5 \mathrm{mM}$ $\mathrm{MgCl}_{2}, 1 \mathrm{mM}$ EGTA (pH 8.0), $0.1 \mathrm{mg} / \mathrm{mL}$ BSA, $0.005 \%$ Tween 20], $100 \mu \mathrm{M}$ dNTPs, 1:25000 SYBR Green I dye, $10 \mathrm{nM}$ ROX reference dye, $1.25 \mathrm{U}$ HotStar Taq polymerase (Qiagen), $0.1 \mu \mathrm{g}$ telomerase substrate primer (a.k.a. 'TS'; 5'-AATCCGTCGAGCAGAGTT-3'; HPLC grade; Invitrogen), and $0.065 \mu \mathrm{g}$ anchored return primer (a.k.a. 'ACX'; 5'-GCGCGG(CTTACC) ${ }_{3}$ CTAACC-3'; HPLC grade; Invitrogen). The reaction mixture was first incubated at $25^{\circ} \mathrm{C}$ for 30 mins to allow the telomerase in the protein extract to elongate the TS primer by adding TTAGGG-repeats. Next, the TRAP reaction was halted and the Taq polymerase was activated by heating at $95^{\circ} \mathrm{C}$ for $15 \mathrm{~min}$. The activation was followed by 40 cycles of $95^{\circ} \mathrm{C}$ for $30 \mathrm{~s}, 60^{\circ} \mathrm{C}$ for $30 \mathrm{~s}$, and $72^{\circ} \mathrm{C}$ for $60 \mathrm{~s}$. Melting curve analysis was automatically carried out after completion of the $40^{\text {th }}$ cycle to verify the amplification specificity. Thermal cycling was conducted using the ABI 7500 Fast Real Time PCR System (Applied Biosystems, California, USA). Samples were run in triplicate. The $\mathrm{C}_{\mathrm{q}}$ values were determined from semi-log amplification plots (log increase in fluorescence signal against cycle number). The relative telomerase activity was calculated by the $2^{-\Delta \mathrm{Cq}}$ method [69].

\section{Sex hormone analysis}

Blood samples were diluted with $300 \mu \mathrm{L}$ double-distilled water and mixed with $2 \mathrm{~mL}$ of diethyl ether (SigmaAldrich). The mixture was vortexed for 10 seconds and centrifuged at $2000 \times \mathrm{g}$ for $10 \mathrm{mins}$. The upper, organic phase was transferred to glass centrifuge tube carefully. The extraction procedure was repeated 3 times to ensure complete recovery of the sex hormones. Diethyl ether was then evaporated under gentle stream of nitrogen gas (Hong Kong Oxygen Ltd, Hong Kong). Concentrations of 
estradiol, testosterones and 11-ketotestosterone were quantified using commercially available enzyme immunoassay kit (Cat.582251, Cat.582701, Cat.582751, respectively; Cayman Chemical, Michigan, USA) following the manufacturer's recommended procedures. Plasma protein concentration of the whole blood was measured by the use of Bradford protein assay (Bio-Rad) and was used to normalize the concentration of sex hormones.

\section{Statistical analyses}

All statistical analyses were performed in the $\mathrm{R}$ environment 2.15.1. Survival probability of male and female medaka across age was estimated using the Kaplan-Meier method adjusted for left-truncation ( $<3$ month-old) and right-censoring (up-to-date survivorship data at the time of this writing). Sex difference in longevity was indicated by contrasting the survival curves of the two sexes using logrank test. The age-dependent TRF length was modeled as exponential decay: $T R F_{t}-$ Plateau $=\left(T R F_{0}-\right.$ Plateau $)$. $e^{-\lambda t}$, where $T R F_{t}$ is the modeled mean TRF length at age $t, T R F_{0}$ is the extrapolated initial mean TRF length, Plateau is the horizontal asymptote, and $\lambda$ is the rate constant. Model parameters were fitted using self-starting nonlinear least squares asymptotic regression model (R/ nls in conjunction with SSasymp function).

Sex-dependency of the telomerase activity and telomeric length were tested using Student's $t$-test. Age-dependent variation in plasma concentration of the three sex hormones' level was illustrated by the use of one-way analysis of variance (ANOVA) followed by Tukey's honestly significant difference (HSD) test. The relationship between telomere length and telomerase activity and the circulatory levels of sex hormones were illustrated by Spearman correlation analyses. Whenever applicable, the normality and homoscedascity assumptions for all parametric inferences were verified by the Shapiro-Wilk test and Levene's test on median center, respectively. $p$-values from multiple comparisons were adjusted to control for false discovery rate (FDR) by use of the Benjamini and Hochberg procedure [70].

\section{Competing interests}

The authors declare that they have no competing interests.

\section{Authors' contributions}

Experimental design: DWTA. Financial support: DWTA. Performed the experiments: GS. Data analysis and interpretation: NKMC, DWTA, GS, and BWPY. Manuscript preparation: GS, NKMC and DWTA. All authors read and approved the final manuscript.

\section{Acknowledgements}

The work described in this paper was supported by grants from the Research Grants Council of the Hong Kong Special Administrative Region, China (Project No. 9041468; CityU 160009), the University Grants Committee, Area of Excellence Grant (AoE/P-04/04) and the State Key Laboratory in Marine Pollution. The authors greatly appreciated Jun Bo, Roy R. Ye, Andy C. K. Cheung and Hong-Lin Ren for their assistance on fish sampling, and Joseph L. Humble for proofreading the manuscript.
Received: 15 September 2013 Accepted: 17 December 2013

Published: 23 December 2013

\section{References}

1. Yip BWP: Relating estradiol and telomeres to longevity in marine medaka Oryzias melastigma. MPhil thesis, City University of Hong Kong, Department of Biology and Chemistry; 2011.

2. Stindl R: Tying it all together: telomeres, sexual size dimorphism and the gender gap in life expectancy. Med Hypotheses 2004, 62:151-154.

3. Aviv A, Shay J, Christensen K, Wright W: The longevity gender gap: are telomeres the explanation? Sci Aging Knowledge Environ 2005, 23:1-16.

4. Cherif H, Tarry JL, Ozanne SE, Hales CN: Ageing and telomeres: a study into organ- and gender-specific telomere shortening. Nucleic Acids Res 2003, 31:1576-1583.

5. Mayer S, Bruderlein S, Perner S, Waibel I, Holdenried A, Ciloglu N, Hasel C, Mattfeldt T, Nielsen KV, Moller P: Sex-specific telomere length profiles and age-dependent erosion dynamics of individual chromosome arms in humans. Cytogenet Genome Res 2006, 112:194-201.

6. Meyne J, Ratliff RL, Moyzis RK: Conservation of the human telomere sequence (TTAGGG)n among vertebrates. Proc Natl Acad Sci USA 1989, 86:7049-7053.

7. Blackburn EH: Telomeres and telomerase: their mechanisms of action and the effects of altering their functions. FEBS Lett 2005, 579:859-862.

8. Harley $C B$, Futcher $A B$, Greider CW: Telomeres shorten during ageing of human fibroblasts. Nature 1990, 345:458-460.

9. Haussmann MF, Mauck RA: Technical Advances: new strategies for telomere-based age estimation. Mol Ecol Resour 2008, 8:264-274.

10. Stewart SA, Ben-Porath I, Carey VJ, O'Connor BF, Hahn WC, Weinberg RA: Erosion of the telomeric single-strand overhang at replicative senescence. Nat Genet 2003, 33:492-496.

11. Kyo S, Takakura M, Kanaya T, Zhuo W, Fujimoto K, Nishio Y, Orimo A, Inoue M: Estrogen activates telomerase. Canc Res 1999, 59:5917-5921.

12. Misiti S, Nanni S, Fontemaggi G, Cong YS, Wen J, Hirte HW, Piaggio G, Sacchi A, Pontecorvi A, Bacchetti S, Farsetti A: Induction of hTERT expression and telomerase activity by estrogens in human ovary epithelium cells. Mol Cell Biol 2000, 20:3764-3771.

13. Simpson ER, Davis SR: Minireview: aromatase and the regulation of estrogen biosynthesis-some new perspectives. Endocrinology 2001, 142:4589-4594.

14. Callard GV, Tarrant AM, Novillo A, Yacci P, Ciaccia L, Vajda S, Chuang GY, Kozakov D, Greytak SR, Sawyer S, Hoover C, Cotter KA: Evolutionary origins of the estrogen signaling system: Insights from amphioxus. J Steroid Biochem Mol Biol 2011, 127:176-188.

15. Viña J, Sastre J, Pallardo F, Borras C: Mitochondrial theory of aging: importance to explain why females live longer than males. Antioxid Redox Sign 2003, 5:549-556.

16. Viña J, Borras C, Gambini J, Sastre J, Pallardo FV: Why females live longer than males: control of longevity by sex hormones. Sci Aging Knowl Environ 2005, 2005:1-17.

17. Viña J, Borras C: Women live longer than men: understanding molecular mechanisms offers opportunities to intervene by using estrogenic compounds. Antioxid Redox Sign 2010, 13:269-278.

18. Allman J, Rosin A, Kumar R, Hasenstaub A: Parenting and survival in anthropoid primates: caretakers live longer. Proc Natl Acad Sci USA 1998, 95:6866-6869

19. Hill K, Boesch C, Goodall J, Pusey A, Williams J, Wrangham R: Mortality rates among wild chimpanzees. J Hum Evol 2001, 40:437-450.

20. Richard AF, Dewar RE, Schwartz M, Ratsirarson J: Life in the slow lane? Demography and life histories of male and female sifaka (Propithecus verreauxi verreauxi). J Zool 2002, 256:421-436.

21. Asdell SA, Doornenbal H, Joshi SR, Sperling GA: The effects of sex steroid hormones upon longevity in rats. J Reprod Fertil 1967, 14:113-120.

22. Austad SN: Postreproductive survival. In Between Zeus and the Salmon, The Biodemography of Longevity. Edited by Wachter KW, Finch CE. Washington, DC: National Academy Press; 1997.

23. Coulson T, Catchpole EA, Albon SD, Morgan BJ, Pemberton JM, CluttonBrock TH, Crawley MJ, Grenfell BT: Age, sex, density, winter weather, and population crashes in Soay sheep. Science 2001, 292:1528-1531.

24. Tinkle DW: The dynamics of a Utah population of Sceloporus undulatus. Herpetologica 1972, 28:351-359. 
25. Ballinger RE, Congdon JD: Population ecology and life history strategy of a montane lizard (Sceloporus scalaris) in southeastern Arizona. J Nat Hist 1981, 15:213-222.

26. Strijbosch H, Creemers RCM: Comparative demography of sympatric populations of Lacerta vivipara and Lacerta agilis. Oecologia 1988, 76:20-26.

27. Ryan MJ, Bartholomew GA, Rand AS: Energetics of reproduction in a neotropical frog, Physalaemus pustulosus. Ecology 1983, 64:1456-1462.

28. Marzona E, Seglie D, Giacoma C: Sexual dimorphism in body size and lifehistory traits in a population of Triturus alpestris alpestris. Ital J Zool 2004, 71:117-120.

29. Lauck B: Life history of the frog Crinia signifera in Tasmania, Australia. Aust J Zool 2005, 53:21-27.

30. Grabherr H, Schabetsberger R, Sztatecsny M: Are sex ratios of larval alpine newts biased in high-altitude spawning sites with different temperature regimes? Amphibia-Reptilia 2009, 30:389-399.

31. Vargas MJ, Sostoa A: Life history of Gambusia holbrooki (Pisces, Poeciliidae) in the Ebro delta (NE Iberian peninsula). Hydrobiologia 1996, 341:215-224.

32. Cabral JAM, João C: Life history, population dynamics and production of eastern mosquitofish, Gambusia holbrooki (Pisces, Poeciliidae), in rice fields of the lower Mondego River Valley, western Portugal. Acta Oecol 1999, 20:607-620

33. Castilho R, Dinis MT, Erzini K: Age and growth of megrim Lepidorhombus boscii, Risso of the Portuguese continental coast. Fish Res 1993 16:339-346.

34. Das GM: Bionomics of the tea red spider, Oligonychus coffeae (Nietner). Bull Entomol Res 1959, 50:265-274.

35. Liles JN, Delong DM: The longevity and productivity of adult male and female Aedes aegypti when reared separately and together on three different diets. Ann Entomol Soc Am 1960, 53:277-280.

36. Scott JA: Lifespan of butterflies. J Res Lepid 1974, 12:225-230.

37. Holldobler B, Wilson EO: The ants. Cambridge: Belknap Press, Harvard University Press; 1990.

38. Lints FA, Bullens P, Le Bourg E: Hypergravity and aging in Drosophila melanogaster: 7. New longevity data. Exp Gerontol 1993, 28:611-615.

39. Carvalho CT, Vasconcellos LEM: Disease, food and reproduction of the maned wolf - Chrysocyon brachyurus (Illiger) (Carnivora, Canidae) in southern Brazil. Rev Bras Zool 1995, 12:627-640.

40. Le Bourg E, Minois N: Failure to confirm increased longevity in Drosophila melanogaster submitted to a food restriction procedure. J Gerontol A Biol Sci Med Sci 1996, 51:B280-B283.

41. Davis T, Wyllie FS, Rokicki MJ, Bagley MC, Kipling D: The role of cellular senescence in Werner syndrome: toward therapeutic intervention in human premature aging. Ann N Y Acad Sci 2007, 1100:455-469.

42. Austad SN: Why women live longer than men: sex differences in longevity. Gender Med 2006, 3:79-92.

43. Maklakov AA, Fricke C, Arnqvist G: Sexual selection affects lifespan and aging in the seed beetle. Aging Cell 2007, 6:739-744.

44. Jemielity S, Kimura M, Parker KM, Parker JD, Cao X, Aviv A, Keller L: Short telomeres in short-lived males: what are the molecular and evolutionary causes? Aging Cell 2007, 6:225-233.

45. Barrett EL, Richardson DS: Sex differences in telomeres and lifespan. Aging Cell 2011, 10:913-921.

46. Terzibasi E, Valenzano DR, Cellerino A: The short-lived fish Nothobranchius furzeri as a new model system for aging studies. Exp Gerontol 2007, 42:81-89.

47. Kipling D, Cooke HJ: Hypervariable ultra-long telomeres in mice. Nature 1990, 347:400-402.

48. Howell A, Clarke RB, Anderson E: Oestrogens, Beatson and endocrine therapy. Endocr-relat Canc 1997, 4:371-380.

49. Russo $\mathrm{H}$, Russo J: Role of hormones in mammary cancer initiation and progression. J Mammary Gland Biol 1998, 3:49-61.

50. Han S, Choi K, Kim J, Ji K, Kim S, Ahn B, Yun J, Choi K, Khim JS, Zhang X, Giesy JP: Endocrine disruption and consequences of chronic exposure to ibuprofen in Japanese medaka (Oryzias latipes) and freshwater cladocerans Daphnia magna and Moina macrocopa. Aquat Toxicol 2010, 98:256-264.

51. Patnaik BK, Mahapatro N, Jena BS: Ageing in fishes. Gerontology 1994 40:113-132.
52. Bolis CL, Piccolella M, Dalla Valle AZ, Rankin JC: Fish as model in pharmacological and biological research. Pharmacol Res 2001, 44:265-280.

53. Gerhard GS: Small laboratory fish as models for aging research. Ageing Res Rev 2007, 6:64-72.

54. Hatakeyama H, Nakamura K, Izumiyama-Shimomura N, Ishii A, Tsuchida S, Takubo K, Ishikawa N: The teleost Oryzias latipes shows telomere shortening with age despite considerable telomerase activity throughout life. Mech Ageing Dev 2008, 129:550-557.

55. Khosla S, Melton $\amalg$ 3rd, Atkinson EJ, O'Fallon WM, Klee GG, Riggs BL: Relationship of serum sex steroid levels and bone turnover markers with bone mineral density in men and women: a key role for bioavailable estrogen. J Clin Endocrinol Metab 1998, 83:2266-2274.

56. Kalu DN: The ovariectomized rat model of postmenopausal bone loss. Bone Miner 1991, 15:175-191

57. Nappi RE, Sinforiani E, Mauri M, Bono G, Polatti F, Nappi G: Memory functioning at menopause: impact of age in ovariectomized women. Gynecol Obstet Invest 1999, 47:29-36.

58. Cong YS, Wright WE, Shay JW: Human telomerase and its regulation. Microbiol Mol Biol Rev 2002, 66:407-425.

59. Soda H, Raymond E, Sharma S, Lawrence R, Davidson K, Oka M, Kohno S, Izbicka E, Von Hoff DD: Effects of androgens on telomerase activity in normal and malignant prostate cells in vitro. Prostate 2000, 43:161-168.

60. Guo C, Armbruster BN, Price DT, Counter CM: In Vivo regulation of hTERT expression and telomerase activity by androgen. J Urol 2003, 170:615-618.

61. Calado RT, Young NS: Telomere diseases. N Engl J Med 2009, 361:2353-2365

62. Counter CM, Hahn WC, Wei W, Caddle SD, Beijersbergen RL, Lansdorp PM, Sedivy JM, Weinberg RA: Dissociation among in vitro telomerase activity, telomere maintenance, and cellular immortalization. Proc Natl Acad Sci USA 1998, 95:14723-14728

63. Harley CB, Liu W, Blasco M, Vera E, Andrews WH, Briggs LA, Raffaele JM: A natural product telomerase activator as part of a health maintenance program. Rejuvenation Res 2011, 14:45-56.

64. Blasco MA, Lee HW, Hande MP, Samper E, Lansdorp PM, DePinho RA, Greider CW: Telomere shortening and tumor formation by mouse cells lacking telomerase RNA. Cell 1997, 91:25-34.

65. Imamura S, Uchiyama J, Koshimizu E, Hanai J, Raftopoulou C, Murphey RD, Bayliss PE, Imai Y, Burns CE, Masutomi K, et al: A non-canonical function of zebrafish telomerase reverse transcriptase is required for developmental hematopoiesis. PLOS ONE 2008, 3:e3364.

66. Ding L, Kuhne WW, Hinton DE, Song J, Dynan WS: Quantifiable biomarkers of normal aging in the Japanese medaka fish (Oryzias latipes). PLOS ONE 2010, 5:e13287.

67. Cheung NK, Hinton DE, Au DW: A high-throughput histoarray for quantitative molecular profiling of multiple, uniformly oriented medaka (Oryzias latipes) embryos. Comp Biochem Phys B 2012, 155:18-25.

68. Kimura M, Stone RC, Hunt SC, Skurnick J, Lu X, Cao X, Harley CB, Aviv A: Measurement of telomere length by the Southern blot analysis of terminal restriction fragment lengths. Nat Protoc 2010, 5:1596-1607.

69. Mok HOL: Relating muscle telomerase to fish growth. MPhil thesis, City University of Hong Kong, Department of Biology and Chemistry; 2008

70. Benjamini $Y$, Hochberg $Y$ : Controlling the false discovery rate: a practical and powerful approach to multiple testing. J R Stat Soc Series B Stat Methodol 1995, 57:289-300.

doi:10.1186/1742-9994-10-78

Cite this article as: Gopalakrishnan et al:: Medaka fish exhibits longevity gender gap, a natural drop in estrogen and telomere shortening during aging: a unique model for studying sex-dependent longevity. Frontiers in Zoology 2013 10:78 